\title{
Making the "inoperable" tumors "operable": Harvey Cushing's contributions to the surgery of posterior fossa tumors
}

\author{
Mahdi Malekpour, M.D., and Aaron A. Cohen-Gadol, M.D., M.Sc. \\ Goodman Campbell Brain and Spine, Department of Neurological Surgery, Indiana University School of \\ Medicine, Indianapolis, Indiana
}

\begin{abstract}
Harvey Cushing played a pivotal role in establishing neurosurgery as a distinct surgical discipline. One of his most important contributions was defining the surgical removal of posterior fossa tumors. Compulsive preoperative evaluation followed by meticulous surgical technique as well as incorporation of maneuvers such as ventricular puncture and electrocautery further advanced resection of tumors in this region. Herein, the authors review Cushing's contributions to posterior fossa surgery.
\end{abstract}

(http://thejns.org/doi/abs/10.3171/2014.2.FOCUS13580)

KEY WoRds • brain neoplasms • Harvey Cushing • neurosurgical procedures

\begin{abstract}
When an experience such as this is now looked back upon, it seems that only stupidity could have so long delayed our better understanding of these lesions.
\end{abstract}

HARvey CUSHING ${ }^{5}$

Harvey Cushing (1869-1939) is considered the father of modern neurosurgery because of his contributions that established it as a distinct discipline. He began his career when neurological surgery was considered a futile endeavor-most cases were deemed inoperable because of their high associated mortality rates. Yet he persisted, pioneering techniques that formed the basis of our distinct specialty. His approach to the posterior fossa is an excellent example of his journey to overcome challenges within the underexplored facet of brain surgery. In 1901, when Cushing returned to Johns Hopkins, surgical decompression to relieve tension was the treatment of choice for most cranial tumors. He believed in neurosurgery as a discipline that would survive the test of time and its critics if the effectiveness of tumor resection could be validated.

In terms of improving outcomes for individuals with brain tumors, accurate and precise localization of the lesion was the first step, as patient selection was, and still is, one of the most important factors in achieving desirable outcomes. Cushing also realized that early diagnosis would improve surgical outcomes. Through his lectures, he therefore attempted to increase the awareness in the medical community of the diagnoses of what were then underrecognized cerebellar tumors. He considered the role of physical examination to be pivotal in evaluating cerebellar growths and building a differential diagnosis (Fig. 1). His notes, patient records, and papers are elaborate descriptions of diverse symptoms that mirror his be- lief in scrupulous documentation. ${ }^{4-6}$ Inasmuch as imaging modalities were merely in their preliminary stages, physical examination to localize any lesion was indispensable. Likewise, any lesion-localizing method would have provided invaluable information at this time. In fact, Cushing pioneered the use of x-ray imaging for diagnosing tumors and planning their excision. ${ }^{2}$ At times, photos of Cushing or his residents showed them holding an x-ray film in their hands, underscoring their utilization of what was then state-of-art technology (Fig. 2).

At around the same time, Dandy (1886-1946) advanced imaging technology further by introducing cerebral pneumoencephalography. Dandy advocated using this modality to diagnose and localize tumors when signs and symptoms had failed to establish the tumor's precise location. ${ }^{8}$ However, Cushing was reluctant to adopt this tool because he believed it would undermine the importance of neurological examination in accurate tumor localization. He also favored using local anesthesia for most neurosurgical procedures, including those that were performed in the posterior fossa. As Cushing stated, "Not only does inhalation narcosis vastly increase the difficulties from extracranial bleeding encountered in the preliminary stages of the operation, but it is highly dangerous from the standpoint of respiratory embarrassment particularly when the symptomatology of one of these fourth ventricles is at all advanced." 5

\section{Preoperative Preparation and Operative Techniques}

A patient was positioned on the operating room table prone on a "cerebellar headholder" (Fig. 3). Anesthesia 


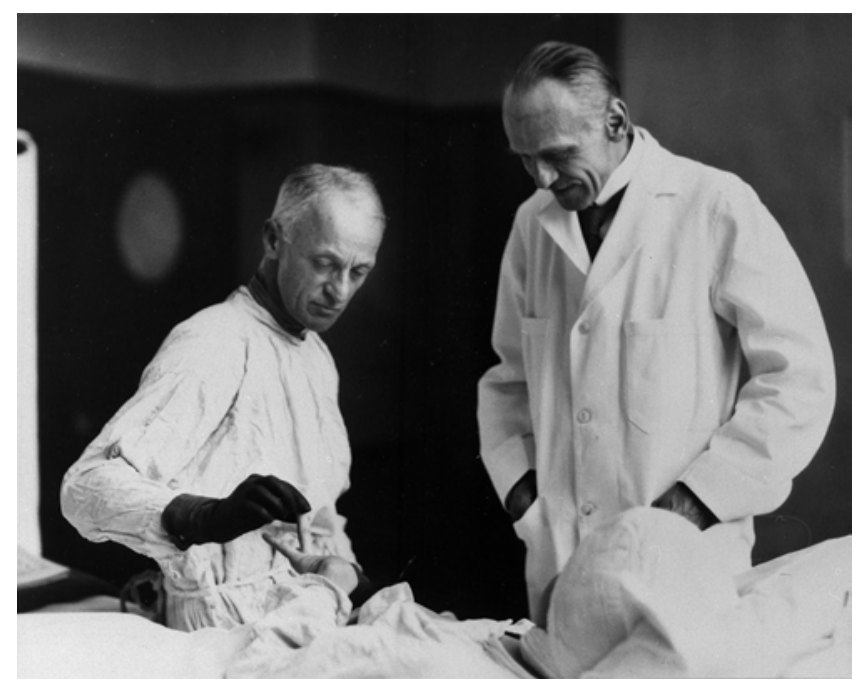

FIG. 1. Cushing performs a postoperative examination on a patient who underwent surgery for a cerebellar tumor. Otfrid Forrester, the visiting surgeon, observes. Courtesy of the Cushing Center at Yale University Department of Neurosurgery.

consisted of a local injection of novocaine and was supplemented by inhalation of ether only if the patient was restless. Cushing avoided ether since he believed it might cause respiratory difficulties, especially among patients with suspicion of brainstem compression. Among his favorite approaches to the posterior fossa was a "crossbow" incision (Fig. 4). This incision provided sufficient room for generous exploration of bilateral posterior fossa contents to find the tumor; at times, he punctured the hemispheres using a needle to look for a cyst or he carefully examined the cerebellar morphology for tumor localization. A crossbow incision also allowed preservation of the blood supply to the scalp flap and facilitated wound healing. This was explained in Cushing's words:

I have found that a vertical median limb, as well as the curved transverse cut, is of advantage in making the exposure.
The vertical intermuscular incision is then carried down to the spines of the upper cervical vertebrae, care being taken to split the ligamentum nuchae, and to separate the deeper muscles in the midline. These primary openings are enlarged with rongeurs, especial care being requisite when approaching the raphe at the midline, the crossing of which may require all of the surgical tricks for the control of hemorrhage from bone and dura that one can summon to his aid. The opening of the bone is enlarged in all directions as far as possible, even upward under the fringe of divided muscle, so as to expose, if it is desirable, the lateral sinus on each side of the torcula. In a downward direction, the opening may be enlarged as far as the foramen magnum. When the bone defect has been increased to the desired size, a transverse incision is then made in the dura on each side of the median occipital sinus which should be carefully approached from each side and ligated. The wound should be closed in layers, and if possible, without drainage. ${ }^{4}$

Cushing advocated the use of this relatively extensive incision because of its superiority in expanding the exposure of the bilateral cerebellar hemispheres. He realized that suboccipital decompression remained an important adjunctive tool in the management of posterior fossa tumors whose gross-total resection, as in cases of acoustic neuromas, was often not deemed possible. He minimized the risk of CSF leakage by employing elaborate meticulous surgical techniques he learned from William Halsted (1852-1922). Cushing wrote that "Unquestionably the bilateral operation requires greater technical skill and is more time consuming. In every cerebellar operation we look forward to 'an hour for exposure and an hour for closure' irrespective of the time."'6

Another step in advancing surgery within the tight space of the posterior fossa was the use of supratentorial ventricular puncture, which decreased tension and allowed safe manipulation within and examination of the posterior fossa structures. ${ }^{3}$ The next major breakthrough was the introduction of electrocautery. Cushing described it as a "surgical trick," and it dramatically accelerated his technical ability to tackle tumors that had been considered inoperable. This tool made its way into the practice of neurosurgery in 1927. The introduction of electrocau-

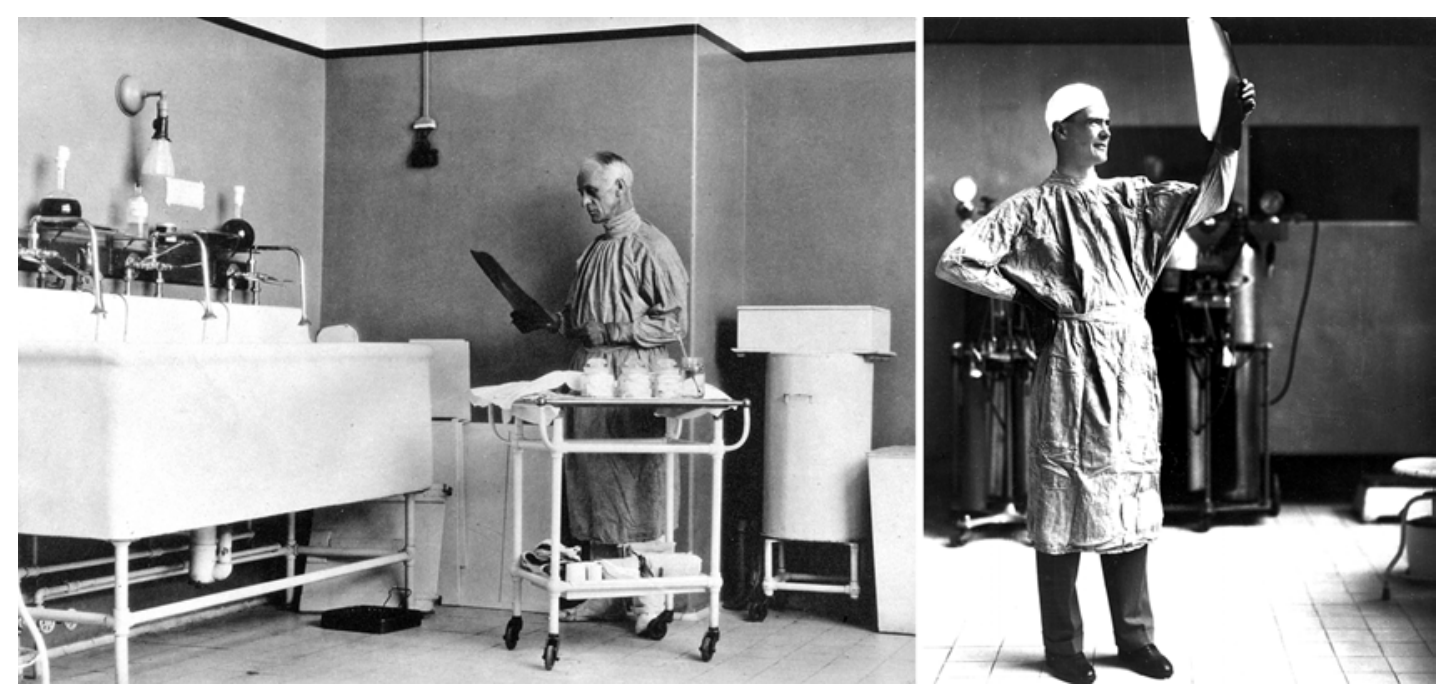

FIG. 2. Photos of Cushing (left) and his resident Norman Dott in 1924 (right) holding up an x-ray film, which provides evidence of their involvement with this technology that was state-of-art at the time. Courtesy of the Cushing Center at Yale University Department of Neurosurgery. 


\section{Harvey Cushing and surgery for posterior fossa tumors}

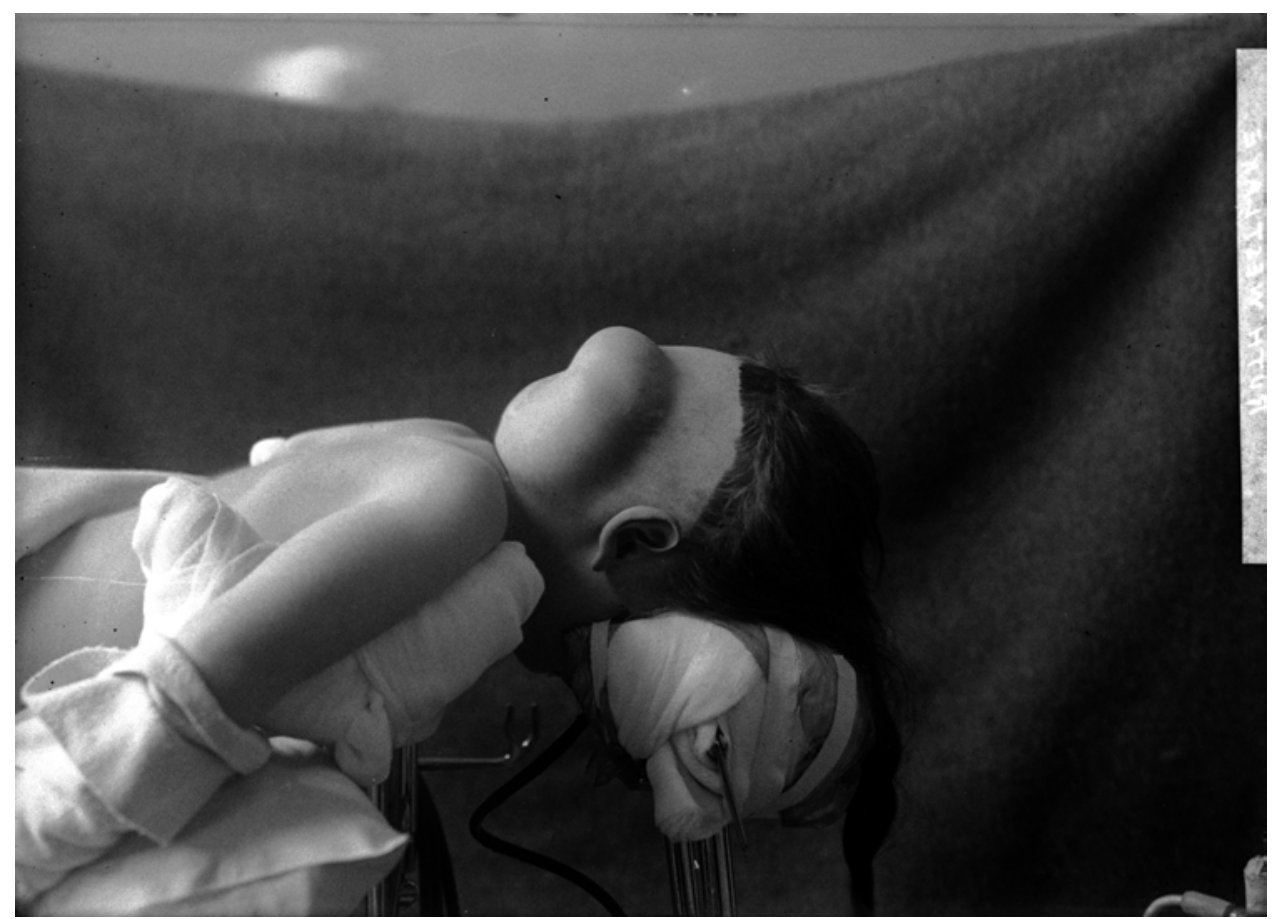

FIG. 3. The patient was positioned on the operating room table prone on a "cerebellar headholder." This patient suffered from a recurrent posterior fossa tumor that had led to a significant bulging and elevations of his "decompression flap." Courtesy of the Cushing Center at Yale University Department of Neurosurgery.

tery led to control of one of the most limiting factors that had defined neurosurgery as a discipline-intraoperative bleeding. Ultimately, electrocautery decreased operative mortality, but initially it led to higher complication rates because of its use in the management of high-risk tumors.

Cushing attempted cyst and tumor nodule resection in almost every case (Fig. 5). In patients with friable masses such as medulloblastomas, he used a suction device that he noted had increased patients' survival threefold, to nearly 17 months. ${ }^{6}$ The controversy regarding removal of cystic lesions is evident in Cushing's notes:

That these xanthochromic cysts were invariably associated with a mural tumor was unknown or was unappreciated, and
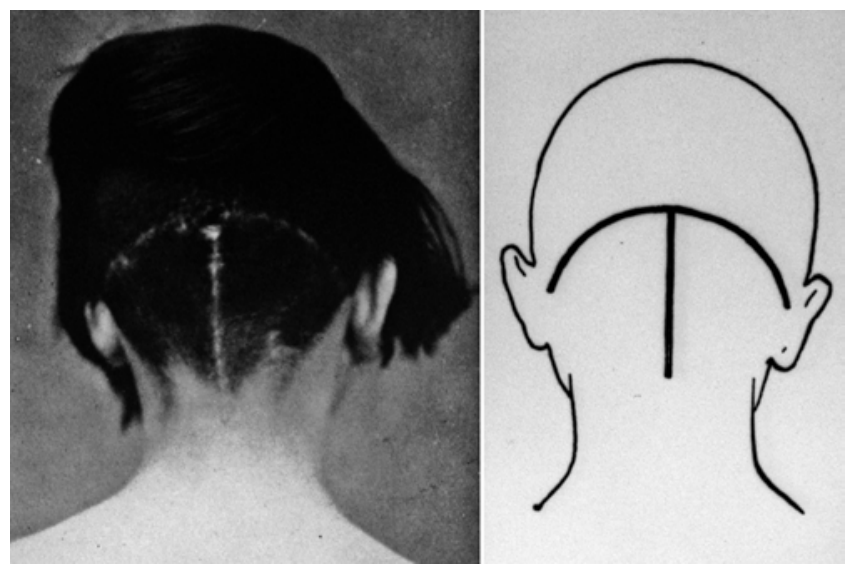

FIG. 4. Cushing's favorite approach to the posterior fossa included a "crossbow" incision. Courtesy of the Cushing Center at Yale University Department of Neurosurgery. the fact that the cyst encountered at the second operation would be found later to have become largely replaced by a solid growth was wholly unforeseen. ${ }^{5}$

This controversy stemmed from the belief that cysts were degenerated gliomas, an opinion advocated by Sir Victor Horsley (1857-1916) before Cushing. Other surgeons typically only punctured the fluid cyst despite identification of a concurrent mural tumor. Cushing commented: "puncturing the lateral cerebellar hemispheres in the hope of striking and evacuating a cyst, their frequent occurrence in the cerebellum having become well known." ${ }^{5}$

When Cushing analyzed his initial experience, he found out that primary cyst aspiration was associated with a high rate of symptomatic cyst recurrence. As he removed part of the mural nodule solely for the purpose of his histological studies, he discovered that patients in whom most of the nodule was removed had lower rates of cyst recurrence. Consequently, in 1922, the significance of mural nodule secretion in maintaining cyst fluid was definitively appreciated, and removal of the nodule became Cushing's standard practice in the subsequent posterior fossa operations. He described his aggressive approach to resection of these nodules in "Experiences with the cerebellar astrocytomas." 5 In his approach, Cushing paid particular attention to the characteristics of tonsillar herniation, prominence of the vermis, and widening of convolutional folia. ${ }^{5}$ He suggested using a midline transection of the vermis when no localizing surface findings were observed, basing this decision on the accuracy of preoperative diagnosis, which was heavily dependent on physical examination. ${ }^{5}$ In most cases, he had identified a deep tumor after cortical transection and described the 

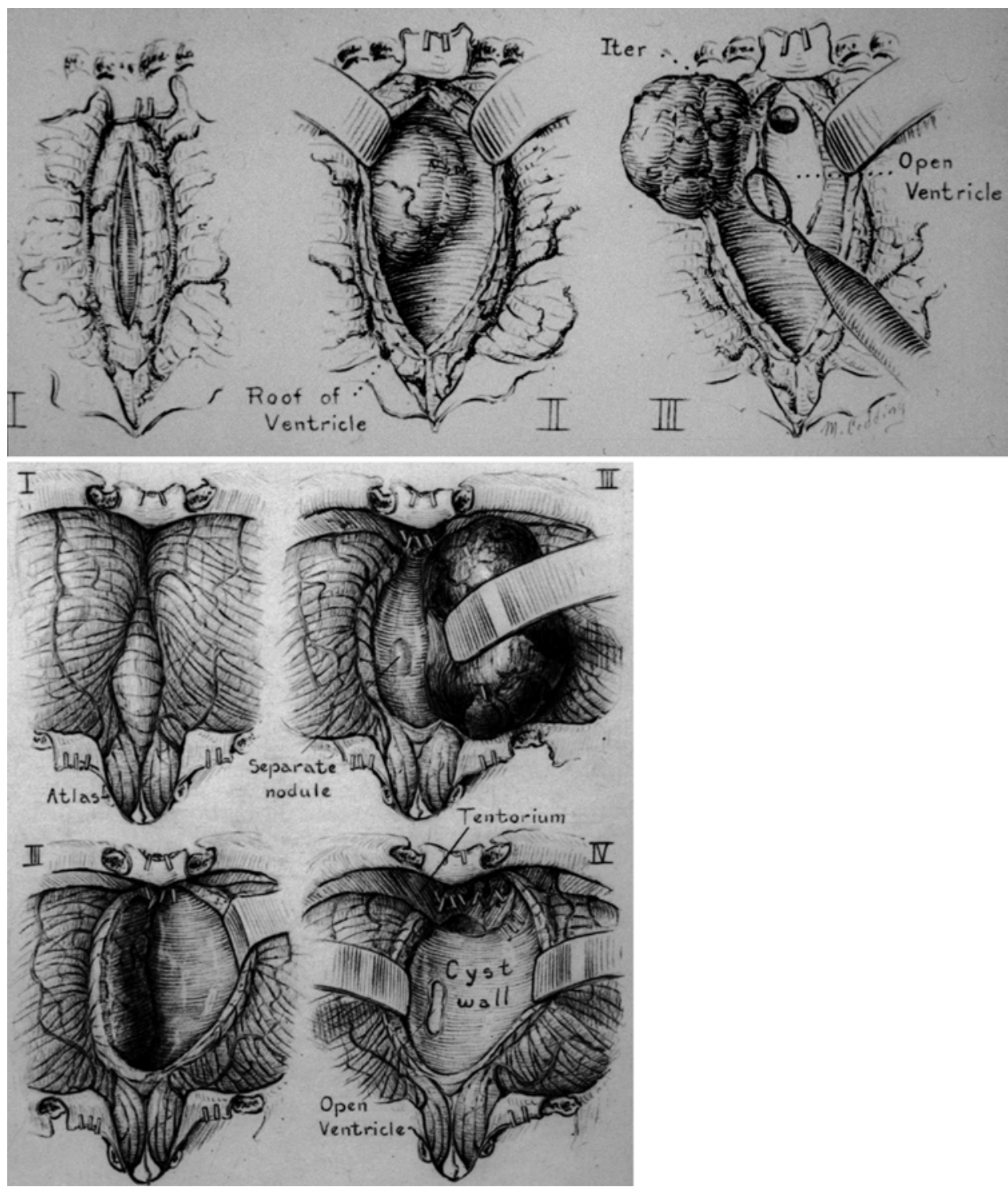

FIG. 5. Cushing used an electrocautery "ring" to achieve gross-total resection of cystic posterior fossa tumors. Maximal removal of the cyst nodule became an important aspect of Cushing's practice later in his career. Courtesy of the Cushing Center at Yale University Department of Neurosurgery.

outcome as follows: "After the removal of huge recurrent cerebellar tumors little may be left but a mere shell of cerebellum, yet it may suffice for all practical purposes of equilibrium and muscle tone." ${ }^{5}$

Cushing understood the importance of postoperative care in advancing operative outcome. He kept the patients prone on the operating room table after surgery for 24 hours. This practice allowed expeditious reopening of the wound, if necessary, and minimized aspiration risk since these patients were often nauseated and might have suffered lower cranial nerve dysfunction. He also used an elaborate "cast" to cover the incision (Fig. 6). He paid special attention to postoperative care. Cushing believed that skilled nurses should take care of patients postoperatively to improve outcome because they can provide:

the undivided attention of someone who is familiar with all the possibilities of accident, who knows when sponging should be resorted to on the threat of hyperthermia, what symptoms suggest the formation of a postoperative clot that will need reopening of the wound, when it is safe to remove the patient from the table to his bed. ${ }^{6}$

Cushing contributed to many other aspects of the care for posterior fossa tumors. Along with his colleague Percival Bailey (1892-1973), Cushing extensively studied the histopathological characteristics of posterior fossa tumors. ${ }^{1,7}$ During his outstanding career, Cushing elevated the treatment of posterior fossa tumors from "palliative care" to therapeutic resection with acceptable risks. He implemented technological breakthroughs that included the use of preoperative imaging, local anesthesia, electrocautery, and postoperative radiation, which as a whole form the basic concept of today's practice of neurosurgical oncology. Figures 7 and 8 include a photograph of a patient and Cushing's operative sketches, which illustrate his practice in relationship to surgery of posterior fossa tumors.

Advances in posterior fossa surgery were also made 


\section{Harvey Cushing and surgery for posterior fossa tumors}
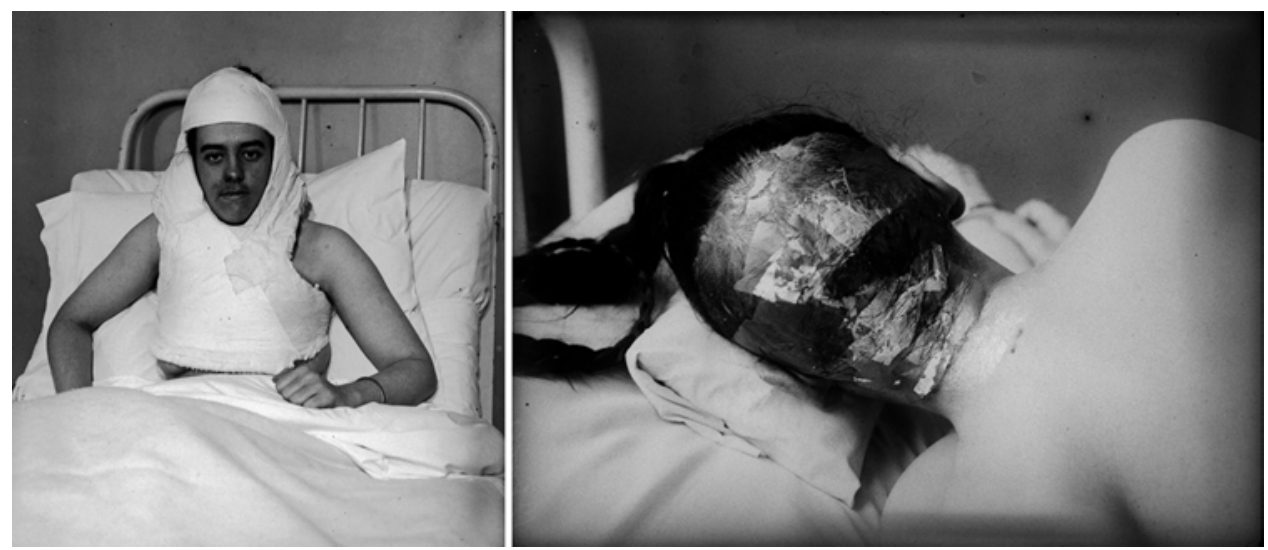

Fig. 6. An elaborate "cast" was used to cover the surgical wound (left), which was further reinforced in layers (right). Courtesy of the Cushing Center at Yale University Department of Neurosurgery.

possible due to contributions of other neurosurgical giants. Fedor Krause (1856-1937) was a German pioneer in neurosurgery who is recognized as the father of neurological surgery in Germany. He described an extradural approach for the treatment of trigeminal neuralgia. He also established an intradural posterior fossa approach to treat tinnitus; he later used this technique to manage acoustic neurinoma. In 1926, Krause published a detailed description of the supracerebellar-subtentorial approach to the third ventricle and pineal gland, which is still widely used. ${ }^{10}$ Charles Harrison Frazier (1870-1936) established neurosurgery in Philadelphia. He worked with William Spiller (1863-1940) at University of Pennsylvania and modified some of Krause's procedures.

French neurological surgery is indebted to Thierry de Martel (1875-1940), Clovis Vincent (1879-1947), and a great neurologist, Joseph Babinski (1857-1932). De Martel was enrolled in an engineering school, which played a pivotal role in developing his interest in neurosurgical instruments and future invention of neurosurgical tools. He wrote about trigeminal neuralgia and cerebellopontine angle tumors, yet his contributions to the development of neurosurgical instruments, including the automatic trephine, are globally appreciated. ${ }^{9}$ Although other contemporary surgeons performed surgery in the region with variable success, Cushing played a major role in refining and improving the outcomes of posterior fossa surgery.

\section{Conclusions}

Harvey Cushing's contributions to surgery in the posterior fossa testify how he overcame some of the most difficult challenges neurosurgery faced in its infancy. Early diagnosis and precise tumor localization, through a detailed history and physical examination, formed one of the cornerstones of his success. He further advanced the field by implementing x-ray imaging and advocating electrocautery, ventricular puncture, and tumor nodule resection. Furthermore, he emphasized premier pre- and postoperative patient care. All these contributions paved the way for advancements in the care of patients with posterior fossa tumors and shaped modern neurosurgery.

\section{Disclosure}

The authors report no conflict of interest concerning the materials or methods used in this study or the findings specified in this paper.

Author contributions to the study and manuscript preparation include the following. Conception and design: both authors. Acquisition of data: both authors. Analysis and interpretation of data:
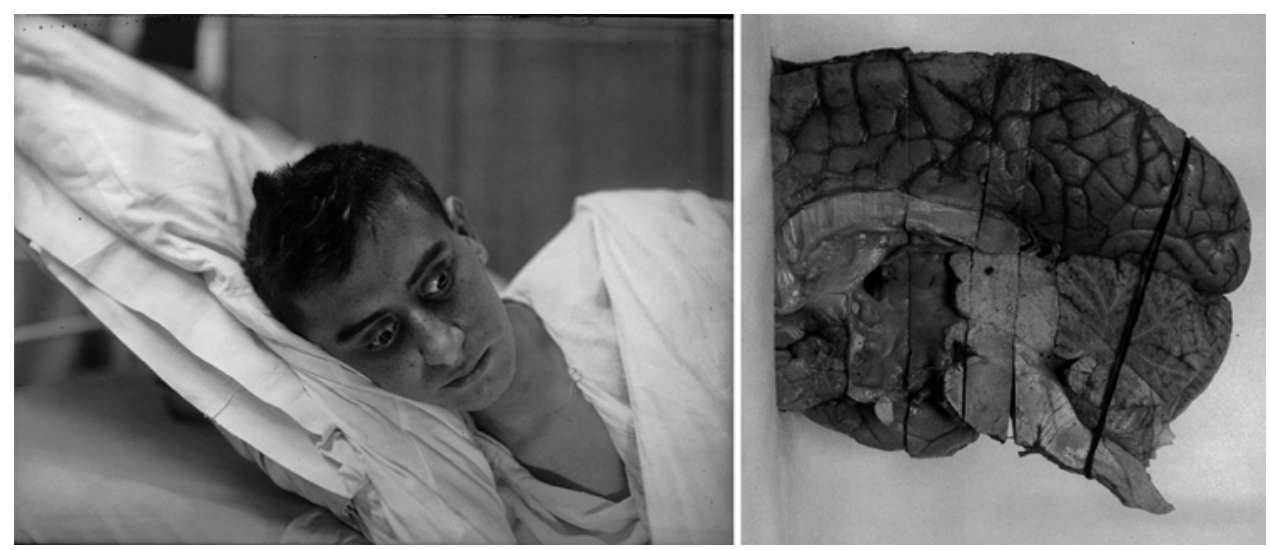

FIG. 7. Left: A young patient presented in 1920 with lethargy and Parinaud's syndrome. The patient expired shortly thereafter. Right: Cushing performed the patient's autopsy, which revealed a pineal region mass. Courtesy of the Cushing Center at Yale University Department of Neurosurgery. 


\section{Malekpour and A. A. Cohen-Gadol}
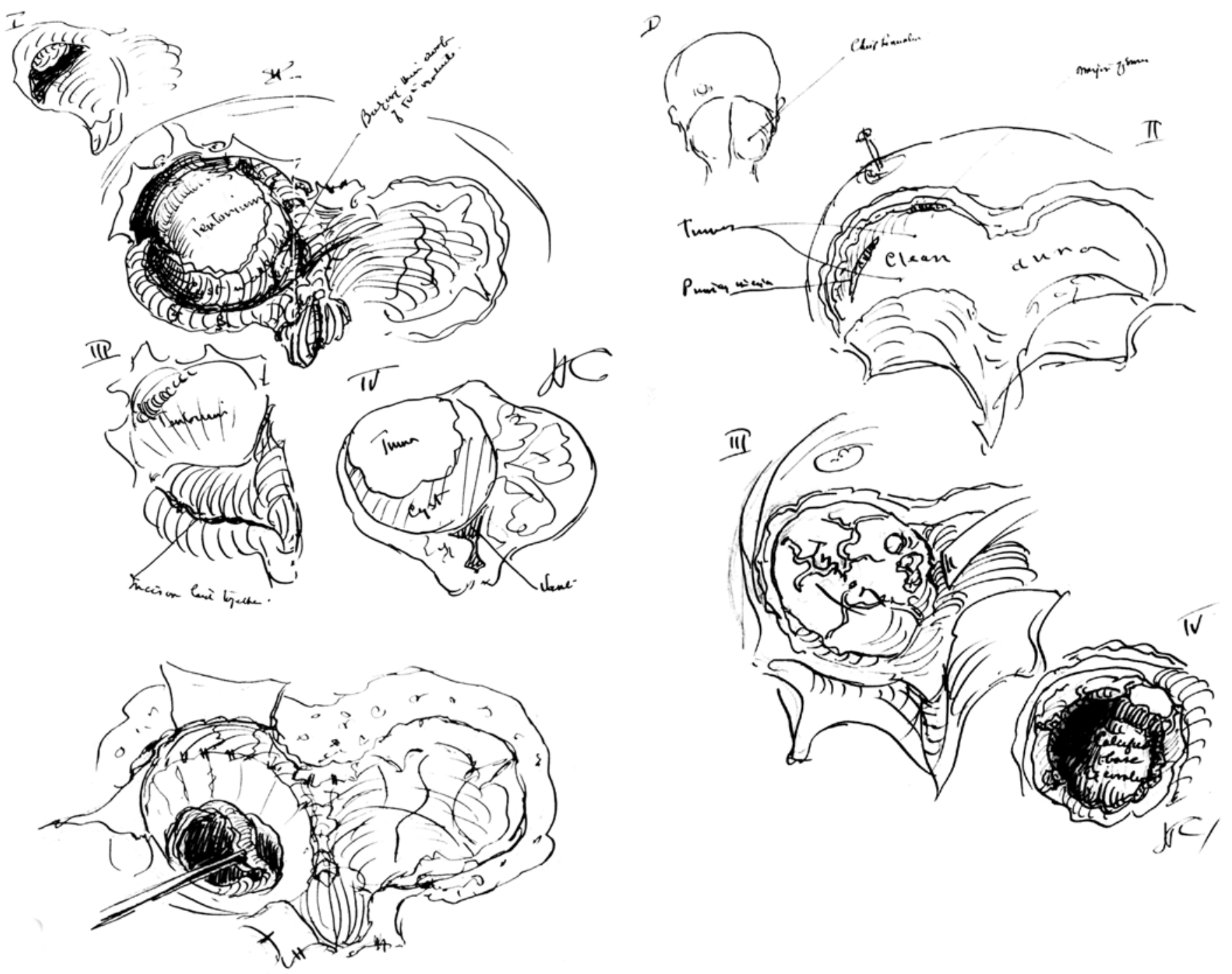

FiG. 8. Cushing documented his intraoperative findings shortly after surgery in elaborate sketches, which illustrate his art in the surgical treatment of posterior fossa tumors. Courtesy of the Cushing Center at Yale University Department of Neurosurgery.

both authors. Drafting the article: both authors. Critically revising the article: both authors. Reviewed submitted version of manuscript: both authors. Approved the final version of the manuscript on behalf of both authors: Cohen-Gadol. Study supervision: Cohen-Gadol.

\section{References}

1. Bailey P, Cushing H: A Classification of the Tumors of the Glioma Group on a Histogenetic Basis With a Correlated Study of Prognosis. Philadelphia: JB Lippincott Co, 1926

2. Bliss M: Harvey Cushing: A Life in Surgery. New York: Oxford University Press, 2005

3. Cohen-Gadol AA, Spencer DD: Inauguration of pediatric neurosurgery by Harvey W. Cushing: his contributions to the surgery of posterior fossa tumors in children. Historical vignette. J Neurosurg 100 (2 Suppl Pediatrics):225-231, 2004

4. Cushing H: The establishment of cerebral hernia as a decompressive measure for inaccessible brain tumors; with the description of intermuscular methods of making the bone defect in temporal and occipital regions. Surg Gynecol Obstet 1:297-314, 1905

5. Cushing H: Experiences with the cerebellar astrocytomas. A critical review of seventy-six cases. Surg Gynecol Obstet 52: 129-204, 1931
6. Cushing H: Experiences with the cerebellar medulloblastomas. A critical review. Acta Pathol Microbiol Scand 7:1-86, 1930

7. Cushing H: The intracranial tumors of preadolescence. Am J Dis Child 33:551-584, 1927

8. Dandy WE: The treatment of brain tumors. JAMA 77:18531859,1921

9. Pecker J: Thierry de Martel. 1875-1940. Surg Neurol 13:401403, 1980

10. Shucart WA: Fedor Krause 1856-1937, in Bucy PC (ed): Neurosurgical Giants: Feet of Clay and Iron. New York: Elsevier, 1984, pp 121-124

Manuscript submitted December 18, 2013.

Accepted February 18, 2014.

Please include this information when citing this paper: DOI: 10.3171/2014.2.FOCUS13580.

Address correspondence to: Aaron A. Cohen-Gadol, M.D., M.Sc., Department of Neurological Surgery, Goodman Campbell Brain and Spine, Indiana University School of Medicine, 355 W. 16th St., Ste. 5100, Indianapolis, IN 46202. email: acohenmd@gmail.com. 